\title{
Analysis and modeling of ASIC area at early-stage design for standard cell library selection
}

\begin{abstract}
Area-delay curve is an effective technique to compare and select the appropriate library at different target delay constraint. However, generating area-delay curve requires timeconsuming synthesis processes. This paper presents a fast area estimation model to allow the designer to select the optimal library for designing area-optimized circuit. The model predicts the area-delay curves for a target circuit based on reduced number of synthesis performed at different frequencies. As compared to the general linear search method, the proposed model with 5 synthesis points results $16.5 \mathrm{X}-18.6 \mathrm{X}$ runtime reduction with average error of 2.74\% 5.74\% in different height libraries implementation. This shows that the proposed model is beneficial for area optimal library selection at the early stage of design.
\end{abstract}

Keyword: Area estimation; Analysis and modeling; ASIC design; Logic synthesis; Standard cell; Cell height 